\title{
A NEW CONCEPT TO IMPROVE MICROWAVE HEATING UNIFORMITY THROUGH DATA-DRIVEN PROCESS MODELLING
}

Jing Zhou, Yingguang Li*, Di Li

National Key Laboratory of Science and Technology on Helicopter Transmission, Nanjing University of Aeronautics and Astronautics, No.29, Yudao Street, Nanjing, China liyingguang@nuaa.edu.cn

Keywords: microwave processing, temperature distribution, heating pattern, neural network

\begin{abstract}
For a long time, the heating pattern of the workpiece within a multimode microwave oven was considered to be highly sophisticated. As a consequence, the uneven microwave heating problem can only be partly alleviated by a random movement between the electromagnetic field and the workpiece, like the rotating turntable or mode stirrers. In this paper, we reported that the heating behavior has a specific correspondence with the power ratio of multiple microwave sources under certain conditions. The influence factors of this relationship and the corresponding mechanisms were systematically studied by both theoretical analysis and experimental investigations. On this basis, a data-driven process model was introduced to learn the material's dynamic temperature behaviors during microwave heating process, and a new concept to improve the microwave heating uniformity by temperature monitoring and active compensation was presented.
\end{abstract}

\section{Introduction}

Microwave energy with the ability to rapidly heat dielectric media has been widely used in domestic and industrial fields [1]. However, the distribution of the electric field in a multimode applicator is usually not uniform, since it is a random combination of all the modes excited in a given frequency range [2]. It is just like the sun shine through the dense woods forming mottled reflections on the ground, without any regularity. In combination with a linear or nonlinear thermal response of dielectric absorption this may result in local thermal runway or cold spots in processed materials [3]. Major problems, such as poor end quality, microbial safety concerns, and overheating, are related to the non-uniform heating process [4].

Up to now, the relationship between temperature behaviors and microwave system settings has been rarely researched in the multimode applicator. Here, microwave system settings are parameters regarding power level, frequency and phase etc., of microwave sources. Traditional model-based method inevitably with a series of approximations and omissions for efficient computation, on the one hand, cannot accurately predict the temperature behaviors under a given boundary condition, especially in the case of heating anisotropic media and multi-scale problems included. On the other hand, the microwave system settings cannot be computed as well after the desired temperature behavior is given. As a result, existing methods attempt to solve the uneven heating problem mainly focused on the relative movement between the electric field and the processed materials. For example, mode stirs and variable-frequency microwave sources [5-6] are designed to change the static electric fields into dynamic ones [7-8], and the turntable is to move the processed object through the areas of high and low power fields alternately [9-10]. 
The above method can, to some extent, improve the temperature uniformity on the surface of the composite parts. However, with a random compensation effect, these methods cannot solve the problem in principle. In this paper, we validated that under certain conditions there is a fixed relationship between the heating behavior and the power ratio of multiple microwave sources from both theoretical analysis and experiment results. The influence factors of this relationship and the corresponding mechanisms were systematically studied as well. On this basis, a data-driven process model was introduced to learn the material's dynamic temperature behaviors during microwave heating process, and a new concept to improve the microwave heating uniformity by temperature monitoring and active compensation was presented.

\section{Experiment}

The microwave oven used in this experiment has an octagonal applicator with two microwave sources on each side, as shown in Fig.1 [11]. For each microwave source, the frequency is stabilized at $2.45 \mathrm{GHz}$ and the power range is $0 \sim 800 \mathrm{~W}$. A fiber optical fluorescence measurement system (Beijing DongFang RuiZe Technology Co., Ltd.) and an infrared thermal imager (FLIR A300) were integrated into the equipment for temperature measurement.

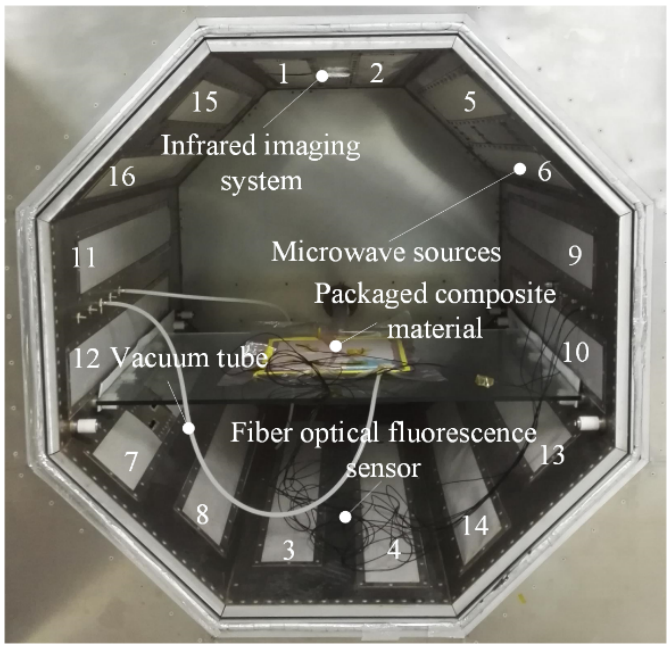

Fig.1 Microwave heating system used in this work.

A $250 \times 250 \times 2.5 \mathrm{~mm}^{3}\left([0]_{25}\right)$ carbon fiber composite laminate (nonmagnetic) was prepared and used as the workpiece in this work. Aluminum foils were stuck on the edge of the laminate to prevent arcing. The carbon fiber reinforced epoxy prepreg (UIN10000), with the thickness of $0.1 \mathrm{~mm}$, was purchased from Weihai Guangwei Composites Co., Ltd. The density and fiber volume fraction of this material were $1560 \mathrm{~kg} / \mathrm{m}^{3}$ and $60 \%$, respectively. The recommended curing temperature was $120^{\circ} \mathrm{C}$.

\section{Results and discussion}

At each time $k, k \geq 0$, any heating process can be described as:

$\mathbf{T}(k+1)=\mathbf{T}(k)+\mathbf{H R}(k) \cdot t_{k}$ 
where $\mathbf{T}(k+1)$ and $\mathbf{T}(k)$ are the temperature distribution at time $k+1$ and $k, \mathbf{H R}(k)$ and $t_{k}$ are the heating rate distribution adopted at time $k$ and its fuction time. Therefore, if $\operatorname{HR}(k)$ can be controlled to compensate the unven $\mathbf{T}(k)$, a uniform microwave heating process can be guaranteed. For convinience, the distribution of the heating rate (or heating rate ratio) on the surface of the workpiece was defined as heating pattern (HP). In order to realize an effective temperature control process, the influence factor of the HP and its influencing mechanism should be studied.

\subsection{Constant HP-MCS relationship in non-transforming materials}

For non-transforming materials (nonmagnetic), the heat transfer model during the microwave heating process can be considered as:

$\rho c_{p} \frac{d T}{d t}=2 \pi f \varepsilon^{\prime \prime} E^{2}+Q_{\mathrm{cd}}+Q_{\mathrm{cv}}+Q_{\mathrm{rd}}$

where $\rho, c_{p}$ and $\varepsilon^{\prime \prime}$ are the density, specific heat capasity and dielectric loss factor of the material; $d T / d t$ is the heating rate; $f$ is the micorwave frequency; $E$ is the electric field strength. $Q_{\mathrm{cd}}, Q_{\mathrm{cv}}$ and $Q_{\mathrm{rd}}$ are the heat flux due to conduction, convection and radiation which usually can be considered as constants or even ignored compared with the absorbed microwave power, when the material properties remain constant. Thus, the HP is directly proportional to the distribution of the absorbed microvave power [11].

To investigate the influence factor of the HP, a large number of heating experiments were carried out under different conditions. Firstly, single microwave source (No.1 to No.16) was used to heat the composite laminate. For each feeding source, the heating experiment was repeated for three times. For each heating process, the maximum temperature was limited below $55^{\circ} \mathrm{C}$ to ensure that the polymerizing reaction of the composite was not triggered and the material properties can be approximated as constants (i.e., non-transforming materials). When the temperature was dropped to room temperature, another heating process was started. Fig. 2 shows the representative HP under the single microwave source. It can be seen that the temperature distribution is extremely uneven where many hot spots and cold spots exist without any regularity. However, the temperature distribution or HP is almost unchanged when the microwave source is confirmed. The above phenomenon can be explained by the following equation which considers the case of a rectangular cavity.

$f_{m n l}=\frac{1}{2 \sqrt{\mu \varepsilon}} \sqrt{\left(\frac{m}{\mathrm{a}}\right)^{2}+\left(\frac{n}{b}\right)^{2}+\left(\frac{l}{d}\right)^{2}}$

where $f_{m n l}$ is the resonant frequency of the electromagnetic modes that can be excited in the microwave cavity; $\mu$ and $\varepsilon$ denote the distribution of the permeability and permittivity for the medium inside the cavity; a,$b$ and $d$ represent the dimension of the cavity in the $x, y$ and $z$ direction; $m, n$ and $l$ are integers which determine the field type of electromagnetic modes. Once the microwave cavity and the object being heated are chosen, the dimension of the cavity (,$b$ and $d$ ), the distribution of the permeability $\mu$ and permittivity $\varepsilon$ are constants, and the field type of electromagnetic modes ( $m, n$ and $l$ ) only depends on the working state of each microwave source including power level, frequency and phase etc. Among them, the control of microwave frequency or phase is very expensive by using traveling-wave tubes and solid-state amplifiers. As an alternative, the power control of microwave sources is a much more economic and feasible way, since most 
industrial microwave heating devices are equipped with multiple magnetrons and it is precisely these equipment that need high heating uniformity. For convenience, the power (or power ratio) of multiple microwave sources are defined as microwave control strategy (MCS). For single microwave source, the electric field directly depends on its location on the resonant cavity. This is the reason why the HP remains unchanged for the same feeding source but was different between different feeding sources (see Fig.2). Additionally, a linear increase in temperature can be observed during each heating process, except for some influence caused by heat dissipation at relatively high temperatures. That is, the heating rate remains constant when the power of the feeding source remains constant, which demonstrates that the effect of the heat conduction, convection and radiation can really be considered as constants or ignored. Considering the space limitation, only the results of No.5 and No.15 microwave source are given in this paper. Similar results were also reported by Sun who used the silica gel membrane (non-transforming) as the material [12].
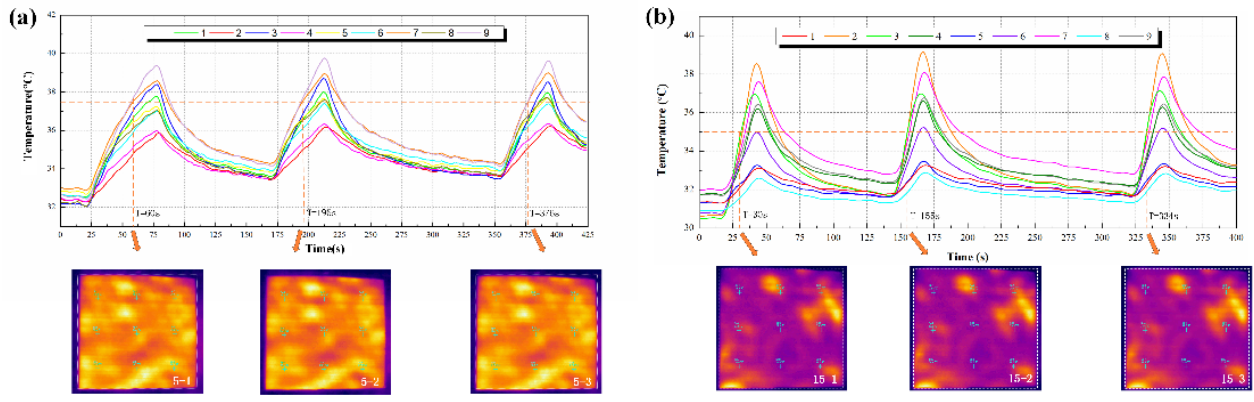

Fig.2 Heating pattern under source No.5 (a) and No.15 (b).

To investigate the situation where multiple sources are included, similar heating experiments with multiple sources were carried out. Representative HP can be found in Fig.3. It can be seen that the result was similar to that of the single source heating experiment. As mentioned above, if the properties of the workpiece remain constant, a fixed HP can be excited by a specific MCS, i.e., $\mathrm{F}(\mathbf{M C S})=\mathbf{H P}$.

In order to further understand whether different feeding sources will interfere with each other, the HP generated by multiple sources was compared with the algebraic sum of the HP under the involved individual feeding source. As shown in Fig.4, various degrees of difference between these two cases can be clearly observed. If only a few feeding sources, for example two or three, work together, the difference between the two curves is small. However, as the number of the feeding source increases, an obvious interference appears between different sources. For instance, a big difference exists in Fig.4 (d) on both the value and the trend between the two curves. This phenomenon can be explained by the power superposition principles. 


\section{High Frequency Heating}

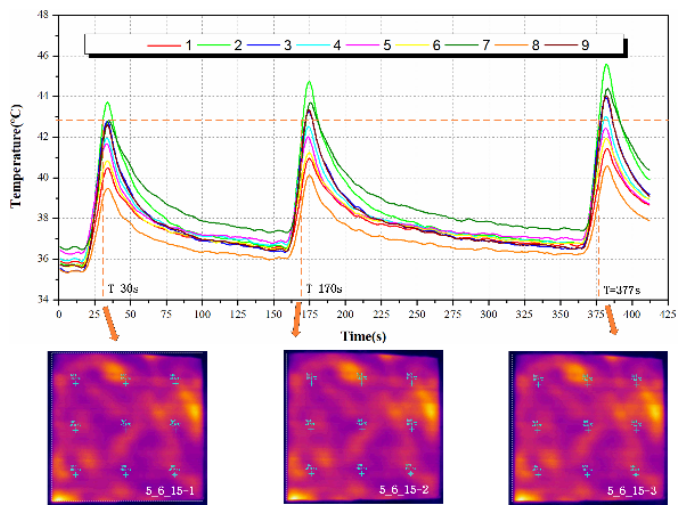

Fig.3 Heating pattern under the combination of source No.5, No.6 and No.15.
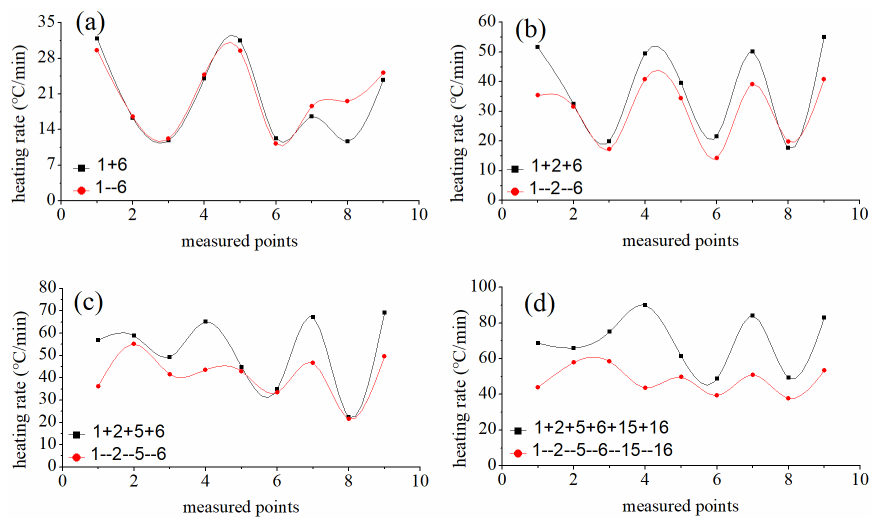

Fig.4 Results of the power superposition experiment. $M--N$ means the HP was generated by source $M$ and source $N$ working together. $M+N$ means the algebraic sum of the HP under source $M$ and source $N$ individually.

Generally speaking, the power superposition principles can be classified into two types, i.e., scalar superposition principle and vector superposition principle. In the first situation, the microwave from different sources is incoherent. Consider the microwave heating system used in this experiment with 16 sources, the total power $P_{i}$ at the point $i$ can be expressed as:

$P_{i} \propto\left|E_{i}^{1}\right|^{2}+\left|E_{i}^{2}\right|^{2}+\mathrm{L}+\left|E_{i}^{16}\right|^{2}$

where $E_{i}^{j}$ is the electrical field provided by source $j$ at the location $i$. In this case, the total power or heating rate at each point on the composite surface is linear with the microwave power provided by each feeding source. This is related to the situation where only a few microwave sources are involved and little probability of interference between microwave sources can be resulted. In the second situation where the microwave from different sources will couple with each other, the total power $P_{i}$ at the point $i$ can be expressed as:

$P_{i} \propto\left|\mathbf{E}_{i}^{1}+\mathbf{E}_{i}^{2}+\mathrm{L}+\mathbf{E}_{i}^{16}\right|^{2}$ 
This clearly indicates that the power superposition is no longer linear, and is more appropriate for the situation where multiple microwave sources are included. As mentioned above, we believe that both the scalar superposition principle and vector superposition principle exist during the microwave heating process. As the number of the operating source increases, the vector superposition contributes more than the scalar one.

It should be noted that in above experiments all microwave sources were worked at the same power level. In the situation where multiple feeding sources work with different power ratios, it can be easily deduced that the HP will vary a lot as well. This is because the magnitude of the electromagnetic mode contributed by each feeding source is inconsistent, although the type and the number of the electromagnetic mode are confirmed once the combination of operating sources is selected.

\subsection{Dynamic HP-MCS relationship in phase change materials}

For phase change materials (nonmagnetic) like polymer composites, the HP will be further influenced by the exothermic curing reaction of the resin matrix.

$\rho c_{p} \frac{d T}{d t}=2 \pi f \varepsilon^{\prime \prime} E^{2}+Q_{\mathrm{cd}}+Q_{\mathrm{cv}}+Q_{\mathrm{rd}}+\rho H_{u} \frac{d \alpha}{d t}$

where $H_{u}$ is the ultimate enthalpy of cure of the composite; $\alpha$ is the degree of cure. In order to investigate how much influence can be resulted during the phase change materials' heating process, a complete microwave curing process was carried out under a fixed MCS. As shown in Fig.5, unlike the heating process within the confined temperature range, an obvious variation in the distribution of the hot spots and cold spots can be observed during the curing process, especially when the temperature of the composite approaches to $120^{\circ} \mathrm{C}$. This is becaused when the curing temperature is reached, the exothermic polymerizing reaction will be triggered for the resin matrix. On the one hand, the released heat will disturb the oringnal HP. On the other hand, basic parameters such as the specific heat capacity, thermal conductivity, permitivitty etc., will change rapidly with the degree of cure in this process.

For the purpose of intuitively reflecting the dynamics during the composite microwave curing process, the curing behaviour of the used composite material was characterized by the differential scanning calorimeter (DSC, Netzsch 204F1), while the specific heat capacity and the thermal conductivity were characterized by TA Q2000 and Netzsch LFA 457. Corresponding results are shown in Fig. 6 which clearly demonstrate that an amount of heat will be released during the composite curing process, accompanying with the variation of some basic properties. What's worse, the change of the material properties will influence the electric field in return according to Eq. 2 and Eq.6. It is these problems that make it almost impossible to accurately predict the HP under a given MCS using the traditional modelbased methods, and vice versa. As mentioned above, the dynamic HP-MCS relationship in phase change materials should be modified to $\mathrm{F}_{\alpha}(\mathbf{M C S})=\mathbf{H P}$. That is, the function $\mathrm{F}_{\alpha}$ holds for a specific value of degree of cure $\alpha$ in a small time interval. 


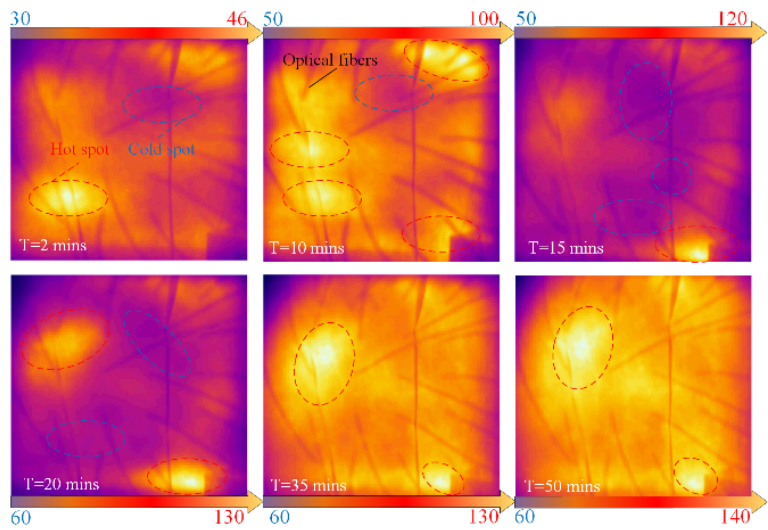

Fig.5 Temperature distribution during the composite microwave curing process under a fixed MCS.
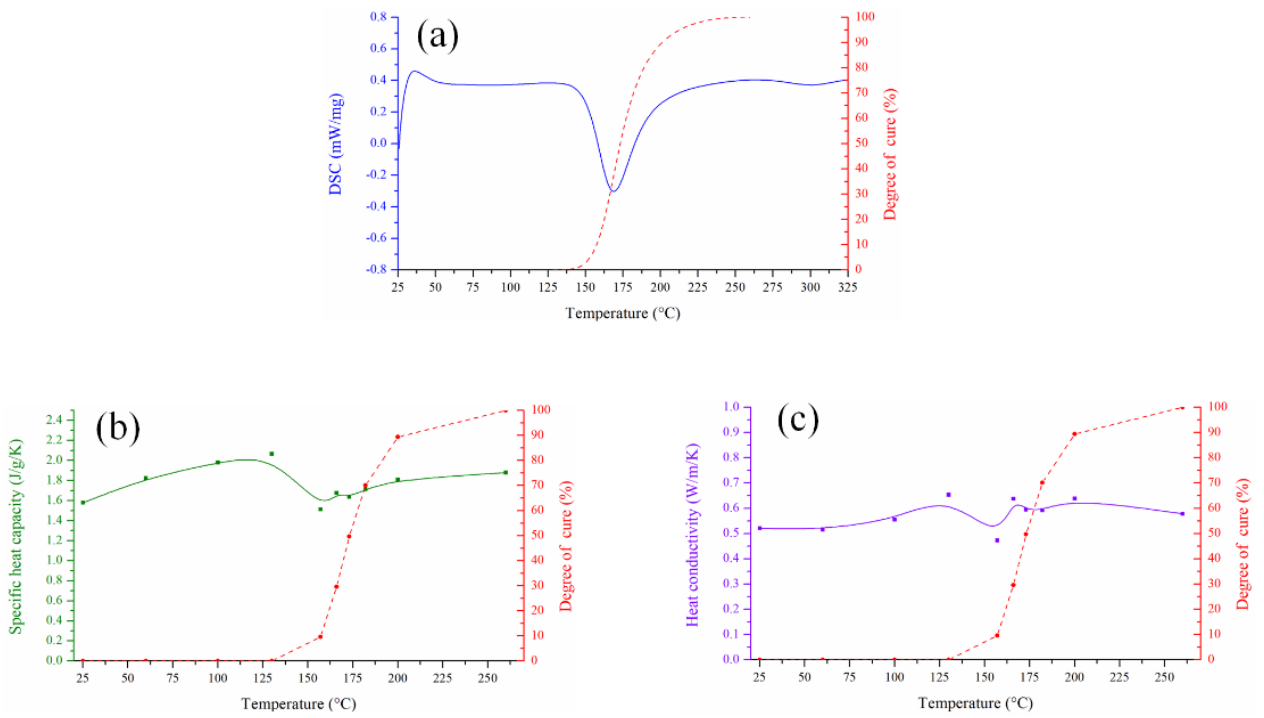

Fig.6 Dynamic properties during the composite curing process: (a) heat generation and variation of degree of cure; (b) variation of the specific heat capacity; (c) variation of the thermal conductivity (perpendicular to fiber direction).

\section{Our solutions for active heating uniformity control}

Based on the above findings, we proposed an online learning based intelligent temperature control method to realize a homogeneous microwave curing process [13]. In this method, a trainable neuron network kept learning the dynamic HP-MCS relationship through analyzing a series of (HP,MCS) datapairs collected online, which continuously provided accurate solutions to the uneven temperature distribution monitored in real time by active compensation. Experimental results indicated that the average temperature difference of a carbon fiber/bismaleimide composite plate $\left(500 \times 400 \times 2 \mathrm{~mm}^{3}\right)$ can be reduced by $\sim 42.3 \%$ compared with existing representative methods. 


\section{Conclusion}

The relationship between HP and MCS was constructed. The influence factors of this relationship and the corresponding mechanisms were systematically studied by both theoretical analysis and experimental investigation. On this basis, a new concept to improve microwave heating uniformity through data-driven process modelling was presented. Our method may pave the way for realizing a homogeneous microwave heating process.

\section{References}

1. Zhong J., Liang S., Yuan Y., et al. IEEE Transactions on Microwave Theory and Techniques, 2016, 64(8), 2467-2477.

2. Domínguez-Tortajada E., Monzó-Cabrera J., Díaz-Morcillo A. IEEE transactions on microwave theory and techniques, 2007, 55(1), 85-91.

3. Link G., Ramopoulos V. Chemical Engineering and Processing-Process Intensification, 2018, 125, 334-342.

4. Vadivambal R., Jayas D S. Food and bioprocess technology, 2010, 3(2), 161-171.

5. H.S.L., Ku T., Yusaf. Progress in Electromagnetics Research B, 2008, 5, 185-205.

6. C. Davis, R. Tanikella, T. Sung, et al. Electronic Components and Technology Conference, 2003 Proceedings, 53 ${ }^{\text {rd }}$, IEEE, 2003, 1718-1723.

7. Plaza-González P., Monzó-Cabrera J., Catalá-Civera J M., et al. IEEE Transactions on Microwave Theory and Techniques, 2005, 53(5), 1699-1706.

8. Sebera V., Nasswettrová A., Nikl K. Drying Technology, 2012, 30(13), 1388-1396.

9. Geedipalli S S R., Rakesh V., Datta A K. Journal of Food Engineering, 2007, 82(3), 359-368.

10. Chen J., Pitchai K., Birla S., et al. Food and bioproducts processing, 2016, 99, 116 127.

11. Zhou J., Li Y., Li N., et al. Composites Part A: Applied Science and Manufacturing, 2018,107,10-20.

12. Sun Y., KIT Scientific Publishing, 2016, 10.5445/KSP/1000051503.

13. Zhou J., Li Y., Li D., Chemical Engineering Journal, 2019, 370, 455-465.

\section{Acknowledgements}

This project was supported by National Science and Technology Major Project of China (Grant no. 2017ZX04002001). 